\title{
Effect of Mach number on Stiffness and Damping Derivatives for Oscillating Hyersonic Non-Planar Wedge
}

\author{
Asha Crasta ${ }^{1}$ and S. A. Khan ${ }^{2}$ \\ 1. Research Scholar, Department of Mathematics, Jain University, Bangalore, Karnataka, India \\ 2. Principal, Department of Mechanical Engineering, Bearys Institute of technology, Mangalore, Karnataka, \\ India
}

\begin{abstract}
A similitude has been obtained for a pitching oscillating Non planar wedge with attached bow shock at high angle of attack in hypersonic flow. A strip theory in which flow at a span wise location is two dimensional and independent of each other is being used. This combines with the similitude to lead to a onedimensional piston theory. Closed form of simple relations is obtained for stiffness and damping derivatives in pitch. The present theory is valid only when the shock wave is attached with the nose of the wedge. With the increase in semi vertex angle of the wedge the stiffness as well as the damping derivatives assumes a high value, and the center of pressure of the wedge also shift towards the trailing edge. Whereas, with the increase in Mach number both stiffness and damping derivative assumes lower values. For higher Mach numbers the stability derivatives become independent of Mach number. From the theory developed some of the results are obtained for wide range of Mach number with remarkable computational ease.
\end{abstract}

Keywords: Hypersonic Flow, Non Planar wedge, Piston Theory, pitch

\section{Introduction}

High incidence hypersonic similitude of Sychev's [1] is applicable to a wing provided it has an extremely small span in addition to small thickness. The unsteady infinite span case has been analyzed, but mostly for small flow deflections. The piston theory of Light hill [2] neglects the effects of secondary wave reflection. Appleton [3] and McIntosh [4] have included these effects. Hui's [5] theory is valid for wedges of arbitrary thickness oscillating with small amplitude provided the bow shock remains attached. Erricsson's [6] theory covers viscous and elastic effects for airfoils with large flow deflection. Orlik-Ruckemann [7] has included viscous effect and Mandl [8] has addressed small surface curvature effect for oscillating thin wedges. Ghosh's [9] similitude and piston theory for the infinite span case with large flow deflection is valid for airfoils with planar or non-planar surfaces whereas Hui's theory [10] is for plane wedges. Ghosh's piston theory has been applied to non-planar cases, both steady and unsteady. The effect of viscosity and secondary wave reflection has not been included. Crasta and Khan have studied the hypersonic and supersonic similitude for planar wedge ([17], [12]), for Delta wing ([11], [13]) and for Delta wing with curved leading edges ([14], [16]). Crasta and Khan have further extended the similitude to study the stability derivatives for Newtonian limit for planar wedge[19], delta wing [18] and delta wing with curved leading edges [15]. In the present work the similitude of Hypersonic planar wedge has been extended for supersonic flows past a non-planar wedge.

\section{Piston Theory}

A thin strip of the wing, parallel to the centerline, can be considered independent of the $\mathrm{z}$ dimension when the velocity component along the $\mathrm{z}$ direction is small. This has been discussed by Ghosh's [9]. The strip theory combined with Ghosh's large incidence similitude leads to the "piston analogy" and pressure P on the surface can be directly related to equivalent piston mach no. $\mathrm{M}_{\mathrm{p}}$. In this case both $\mathrm{Mp}$ and flow deflections are permitted to be large. Hence light hill piston theory [2] or miles strong shock piston theory cannot be used but Ghosh's piston theory will be applicable.

$$
\frac{P}{P_{\infty}}=1+A M_{P}{ }^{2}+A M_{P}\left(B+M_{P}{ }^{2}\right)^{\frac{1}{2}} \quad \text {, Where } P_{\infty} \text { is free stream pressure }
$$

Since strips at different span wise location are assumed independent of each other, the strip can be considered as a flat plate at an angle of attack. The angle of incidence is same as that of wing. Angle $\phi$ is the angle between the shock and the strip. A piston theory which has been used in equation (1) has been extended to supersonic flow. The expression is given below.

$$
\frac{p}{p_{\infty}}=1+A\left(\frac{M_{p}}{\cos \phi}\right)^{2}+A\left(\frac{M_{p}}{\cos \phi}\right)\left(B+\left(\frac{M_{p}}{\cos \phi}\right)^{2}\right)^{\frac{1}{2}}
$$


Where $p_{\infty}$ is free stream pressure, $A=\frac{(\gamma+1)}{4}, B=\left(4 /(\gamma+1)^{2}, \gamma\right.$ is the specific heat ratio and $M_{p}=$ the local piston Mach number normal to the wedge surface.

\section{Pitching moment derivatives}

Let the mean incidence be $\alpha_{0}$ for the wing oscillating in pitch with small frequency and amplitude about an axis $\mathrm{X}_{0}$. The piston velocity and hence pressure on the windward surface remains constant on a span wise strip of length $2 \mathrm{~L}$ at $\mathrm{x}$, the pressure on the lee surface is assumed zero. Therefore, the nose up moment is

$m=-\int_{0}^{L} p . .\left(x-x_{0}\right) d x$

A non planar wedge is obtained by superimposing parabolic arcs on the two sides of the plane wedge.

Therefore The Stiffness and damping derivatives are respectively

$$
\begin{gathered}
-C_{m_{\alpha}}=\frac{2}{\frac{1}{2} \rho_{\infty} U \infty^{2} L^{2}}\left(-\frac{\partial m}{\partial \alpha}\right)_{\alpha=\alpha_{0}, q=0} \\
-C_{m_{q}}=\frac{2}{\frac{1}{2} \rho_{\infty} U \infty^{2} L^{3}}\left(-\frac{\partial m}{\partial q}\right)_{\alpha=\alpha_{0}, q=0}
\end{gathered}
$$

Where $\rho_{\infty}, a_{\infty}$ are density and velocity of sound in the free stream Combining (1) through (5), differentiation under the integral sign is performed. Therefore

$$
-C_{m_{\alpha_{0}}}=\frac{p_{\infty} \cdot A}{\frac{1}{2} \rho_{\infty} U_{\infty}^{2} L^{2} \operatorname{Cos}^{2} \alpha_{0}} \int_{0}^{L}\left(x-x_{0}\right) \cdot \frac{\partial p}{\partial \alpha} \cdot d x
$$

And

$$
-C_{m_{q}}=\frac{p_{\infty} \cdot A}{\frac{1}{2} \rho_{\infty} U_{\infty} L^{3} \operatorname{Cos}^{3} \alpha_{0}} \int_{0}^{L}\left(x-x_{0}\right) \cdot \frac{\partial p}{\partial q} \cdot d x
$$

On Simplifying we get,

The Stiffness and damping derivative are given by

$$
\begin{gathered}
-C_{m_{\alpha}}=\frac{\gamma+1}{2 M_{\infty}^{2} \cos ^{2} \alpha_{0}}\left(I_{1}+I_{2}+I_{3}\right) \\
\text { And }-C_{m_{q}}=\frac{\gamma+1}{2 M_{\infty} \cos ^{3} \alpha_{0}}\left(J_{1}+J_{2}+J_{3}\right)
\end{gathered}
$$

Where 


$$
\begin{aligned}
& I_{1}=M_{\infty}^{2}\left[H \sin 2 \alpha_{0}-\frac{2 \lambda \cos 2 \alpha_{0}}{3}\right] \\
& I_{2}=\left[\left\{-M_{\infty} \frac{(z)^{\frac{3}{2}}}{3 b}\right\}\left\{H \cos \alpha_{0}-\left(H \lambda \sin \alpha_{0}+\cos \alpha_{0}\right)\left(\frac{5 a-3 z}{5 b}\right)+\lambda \sin \alpha_{0}\left(\frac{15 z^{2}-42 a z+35 a^{2}}{3 b^{2}}\right)\right]\right]_{z=a+b}^{z=a-b} \\
& I_{3}=\left[\left\{\frac{M_{\infty} z^{\frac{1}{2}}}{2 \lambda \cos \alpha_{0}}\right\}\left\{-H \sin \alpha_{0} \cos \alpha_{0}+\left(\begin{array}{l}
\left.\left(\sin \alpha_{0} \cos \alpha_{0}-\lambda H\left(\cos ^{2} \alpha_{0}+\cos 2 \alpha_{0}\right)\right)\left(\frac{z-3 a}{3 b}\right)\right) \\
+\lambda\left(\cos ^{2} \alpha_{0}+\cos 2 \alpha_{0}\right)\left(\frac{3 z^{2}-10 a z+15 a^{2}}{15 b^{2}}\right)
\end{array}\right)\right\}\right]_{z=a+b}^{z=a-b} \\
& J_{1}=M_{\infty}\left[H^{2} \sin \alpha_{0}+\left(\frac{\sin \alpha_{0}-2 H \lambda \cos \alpha_{0}}{3}\right)\right] \\
& J_{2}=\frac{-2 z^{\frac{3}{2}}}{3 b}\left[\frac{H^{2}}{4}+H\left(\frac{5 a-3 z}{10 b}\right)+\frac{15 z^{2}-42 a z+35 a^{2}}{140 b^{2}}\right]_{z=a+b}^{z=a-b} \\
& J_{3}=\left\{\left\{\frac{-z^{\frac{1}{2}}}{\lambda \cos \alpha_{0}}\right\}\left\{\begin{array}{l}
\left(\frac{H^{2} \sin \alpha_{0}}{4}\right)-H\left(\sin \alpha_{0}-\lambda H \cos \alpha_{0}\right)\left(\frac{z-3 a}{6 b}\right) \\
+\left(\sin \alpha_{0}-4 \lambda H \cos \alpha_{0}\right)\left(\frac{3 z^{2}-10 a z+15 a^{2}}{60 b^{2}}\right) \\
+\lambda \cos \alpha_{0}\left(\frac{5 z^{3}-21 z^{2} a+35 z a^{2}-35 a^{3}}{70 b^{3}}\right)
\end{array}\right\}\right]_{z=a+b}^{z=a-b}
\end{aligned}
$$

Where

$$
\begin{aligned}
H & =1-2 h \cos ^{2} \alpha_{0} \\
\mathrm{a} & =\left(\frac{4}{\gamma+1}\right)^{2}+M^{2} \sin ^{2} \alpha_{O}, \\
\mathrm{~b} & =\lambda \mathrm{M}^{2} \sin ^{2} \alpha_{\mathrm{O}}
\end{aligned}
$$

Some of the results have been obtained for various Mach Numbers and studied. 
III. RESULTS AND DISCUSSIONS

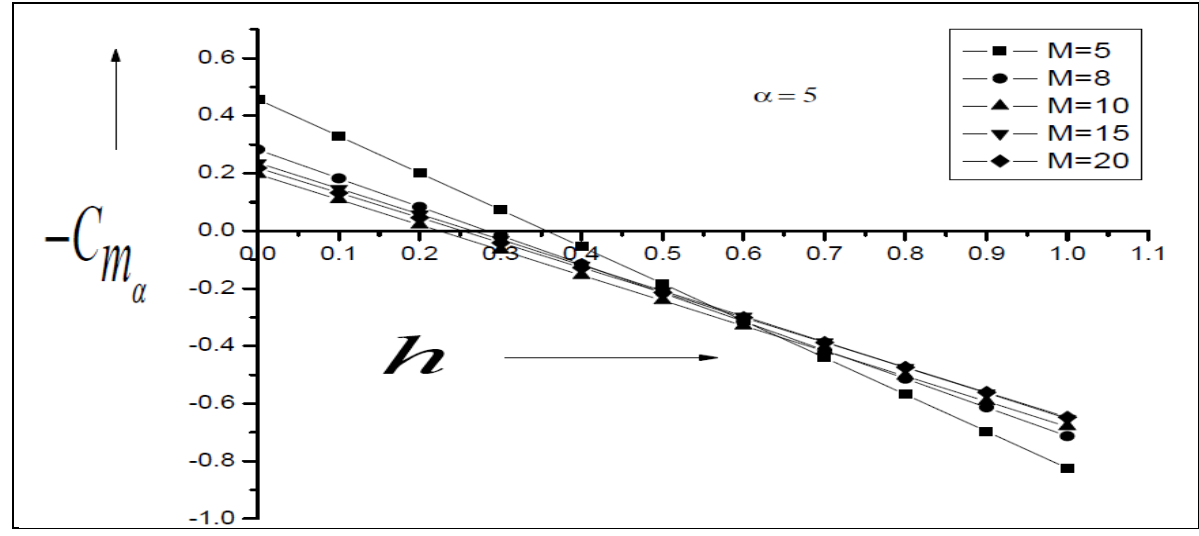

Fig 1: Variation of Stiffness derivative with pivot position

Fig. 1 shows the variation of stiffness derivative with respect to pivot position for different Mach number while the semi vertex angle is kept constant $\theta=5^{0}$. The variation of centre of pressure is 20 to $35 \%$ for Mach number 5, 8, 10,15 and 20 respectively from the leading edge of the wedge. It is also seen from the figure that with the increase in the Mach number there is continuous decrease in the value of the stiffness derivative. Which means with the increase in Mach number there is progressive decrease in stiffness derivative indicating continuous decrease in the static margin which; may not be desirable in most of the cases. However, in the case of fighter planes this is very much needed to have static margin as low as possible to have enhanced maneuverability of the aircraft. Since the semi vertex angle of the wedge is only five degrees. The wedge falls under the category of slender body with a very high length to diameter ratio. In this particular case the shock wave angle will be were small compared to that for semi vertex angle in the range of 20 degree and above. For very high value of semi vertex angle the shock may not be attached with body. Instead of oblique shock there will be a bow shock at the nose of the Non-planar wedge. Due to the presence of bow shock the flow behind the shock will subsonic and flow separation will take place. The present theory is valid only when the shock wave is attached with body (i. e. at the nose of the wedge). Further, the present theory is quasi steady whereas the theory of Hui \& Lui is fully unsteady which more accurate. This difference is observed only in the case of damping derivatives (Fig 2). This is also observed that the magnitude of the damping derivative decreases continuously and this decrease is non-linear, and further, it is found that the center of pressure of the wedge has shifted towards the nose of the wedge. The values of the damping derivatives at the extreme end from the nose are very high as compared to the values in the nose portion of the wedge and this shift in the values may be due the pressure distribution on the wedge surface. Normally the location of the center of pressure will be in the range 50 to 60 percent from the nose and this shift will the system statically as well as dynamically unstable as it was seen as above earlier that with the increase in the Mach number there is substantial decrease in the stiffness derivative.

Fig. 3 shows the variation of stiffness derivative with respect to pivot position for different Mach number while the semi vertex angle is kept constant $\theta=10^{\circ}$. The variation of centre of pressure is 35 to $40 \%$ for Mach number 5, 8, 10, 15 and 20 respectively from the leading edge of the wedge. It is also seen from the figure that with the increase in the Mach number there is continuous decrease in the value of the stiffness derivative as it was seen in Fig. 1, however, the magnitude has increased by 50 percent when semi vertex angle is increased from five degrees to ten degrees. Which means with the increase in Mach number there is progressive decrease in stiffness derivative indicating continuous decrease in the static margin which; may not be desirable in most of the cases. This decrease in stiffness derivative is around fifty percent for the Mach numbers between $M=5$ to 8 and for the rest of the Mach numbers the decrease in the stiffness derivative are marginal. This trend may be due to the Mach number effect. 


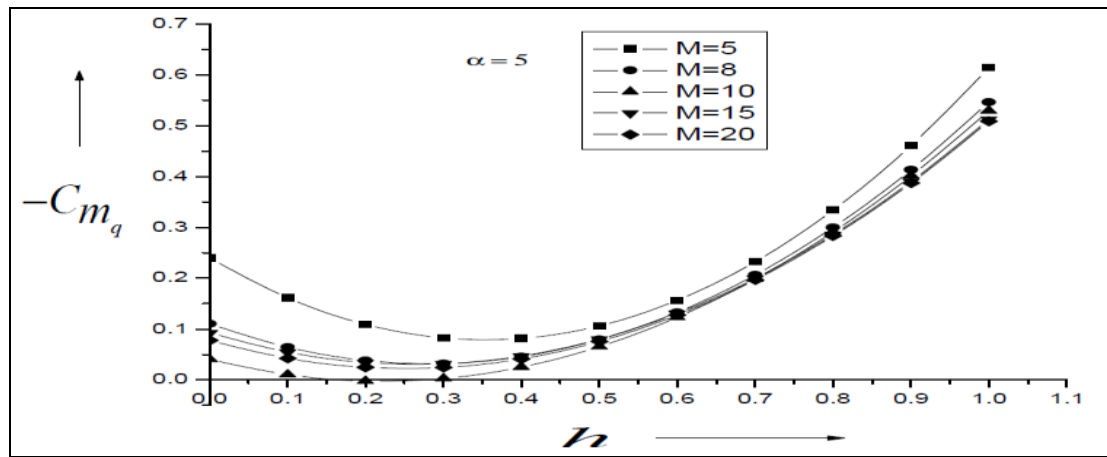

Fig. 2: Variation of damping derivative with pivot position

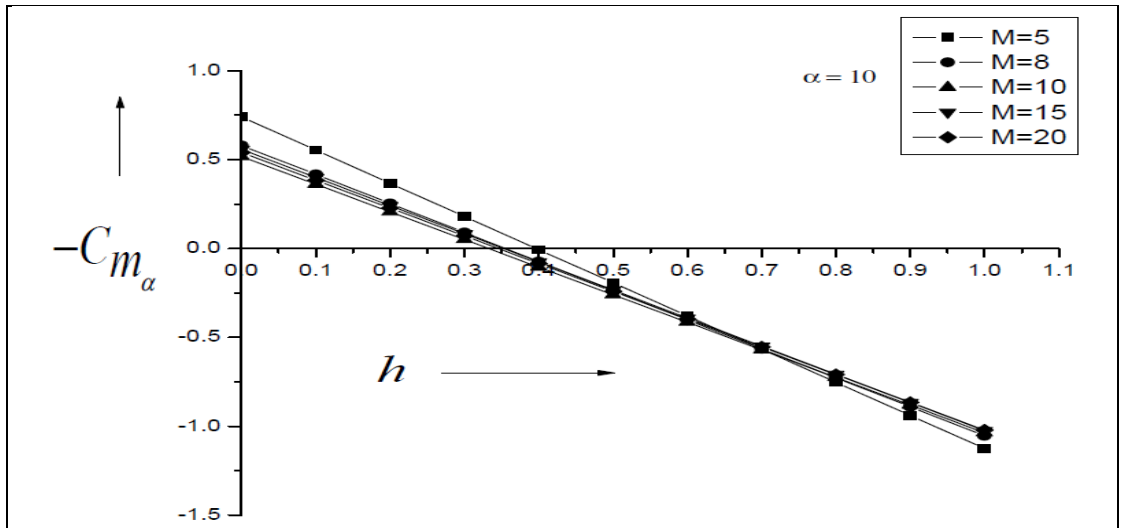

Fig. 3: Variation of Stiffness derivative with pivot position

Fig. 4 presents the results for damping derivatives for semi vertex angle ten degrees. Here once again it is found that there is substantial increase in the damping derivative due to the increase in the semi vertex angle of the wedge, also it is seen that with the increase in the Mach number from five to eight there is sudden drop in the value of the damping derivative, further, increase in the Mach number results marginal decrease in the damping derivative. Due to the increase in the semi vertex angle there is shift in the center of pressure of the wedge towards the aft position of the wedge resulting in the higher values of stability derivatives. It is also observed that the values of the damping derivatives in downstream of the wedge specially for in and around $\mathrm{h}=$ 1 it assumes very high value.

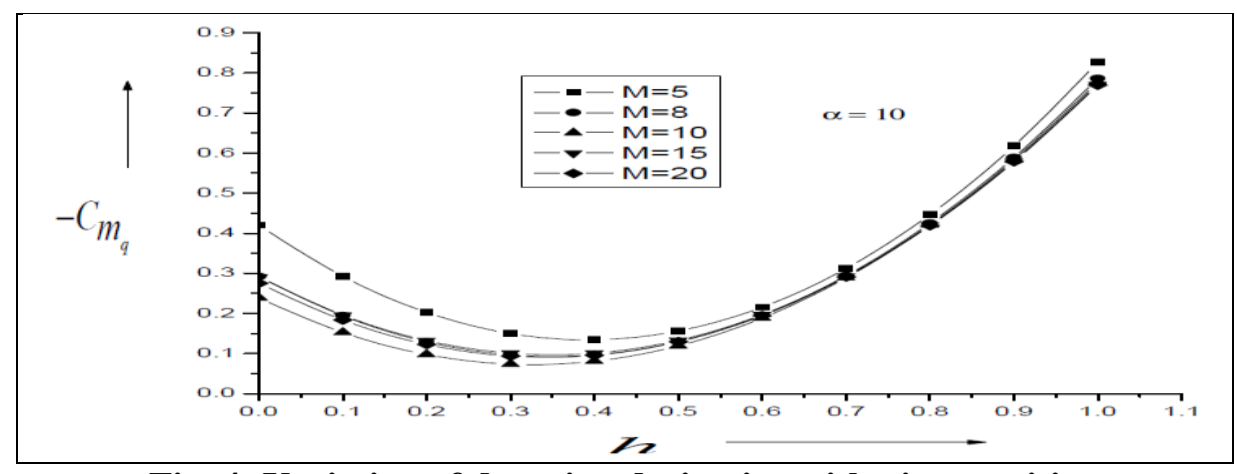

Fig. 4: Variation of damping derivative with pivot position

Fig. 5 presents the stiffness derivatives for semi vertex angle fifteen degrees. From the figure it is found that there is thirty percent increase in the value of the stiffness derivative, whereas, when the Mach number is increased from five to twenty the variation in the stiffness derivative is marginal indicating there is no further, change in the values and the Mach number independence principle hold for the higher Mach numbers. The center of pressure has shifted further downstream in the range from 40 to $45 \%$ from the nose. This may be considered one of the advantages from the static stability point of view. Fig. 6 presents the damping derivatives for semi vertex angle fifteen degrees; it is found that there is over all increase in the value however, with the 
increase in Mach number the variation the damping derivative is only marginal and the center of pressure remains at $\mathrm{h}=0.4$ for the all the values of Mach numbers.

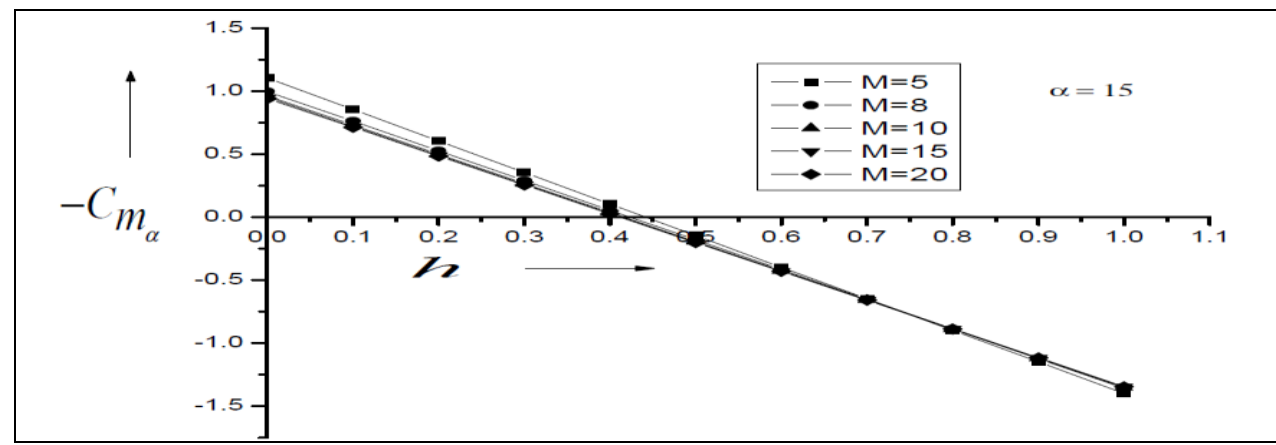

Fig. 5: Variation of Stiffness derivative with pivot position

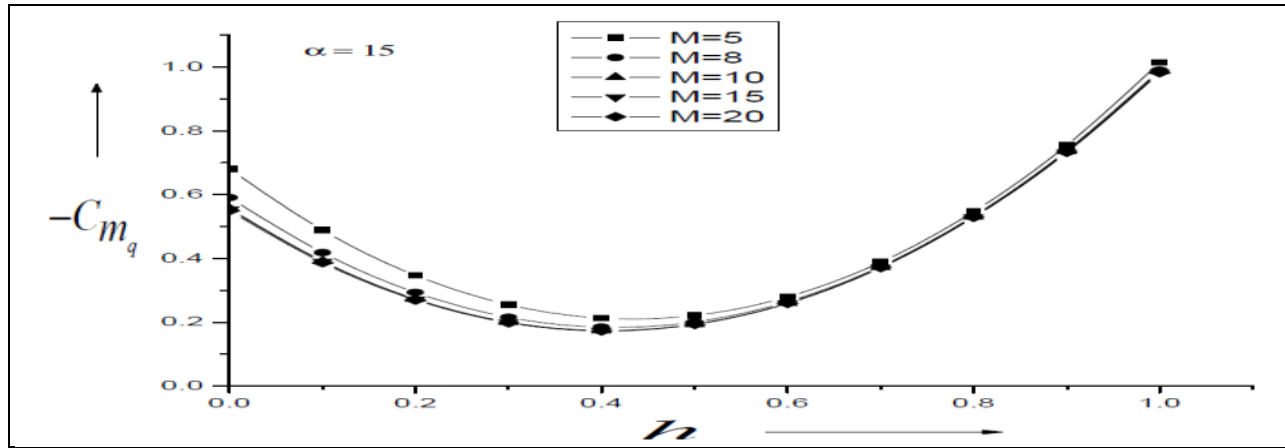

Fig. 6: Variation of damping derivative with pivot position

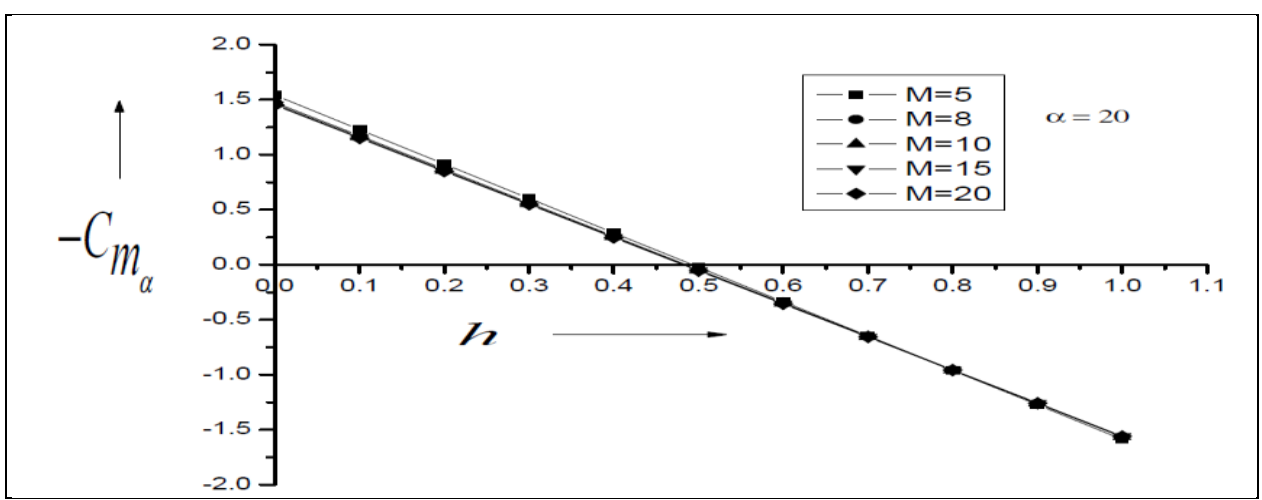

Fig. 7: Variation of Stiffness derivative with pivot position

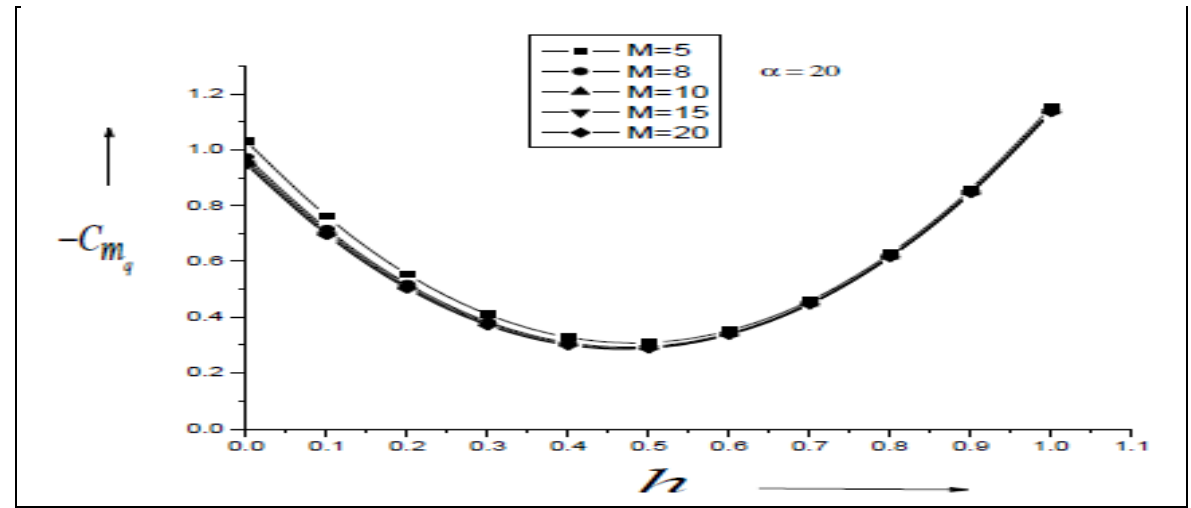

Fig. 8: Variation of damping derivative with pivot position 


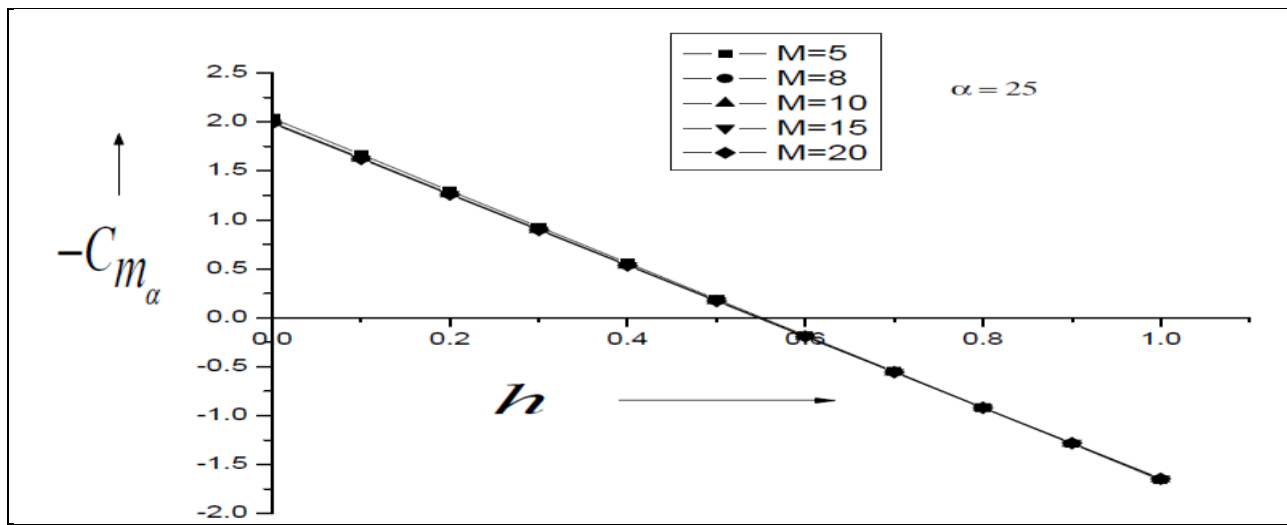

Fig. 9: Variation of Stiffness derivative with pivot position

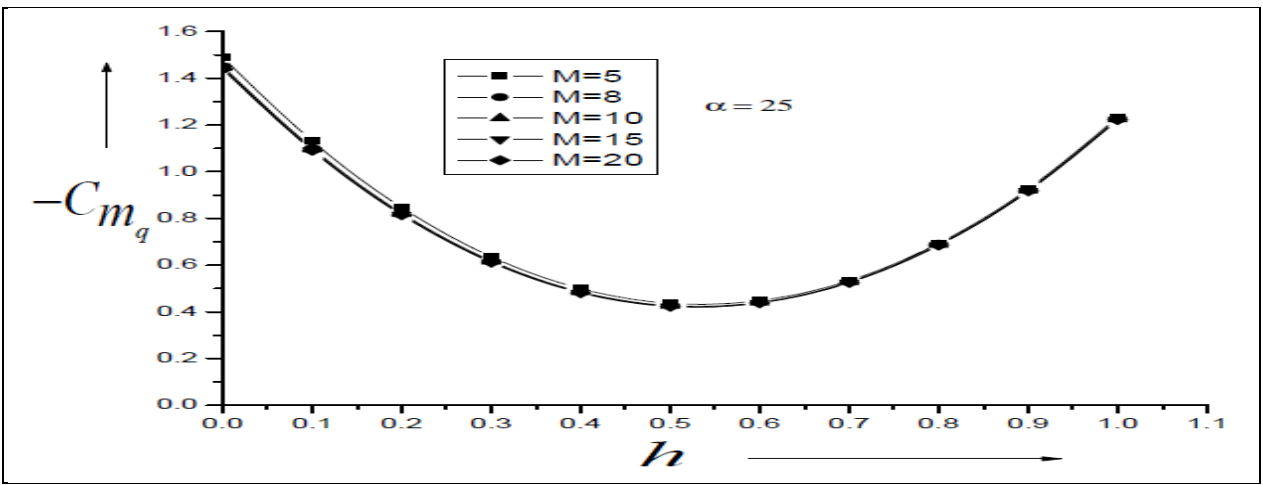

Fig. 10: Variation of damping derivative with pivot position

Results for stiffness and damping derivatives are shown in Figs. 7 to 10 for hypersonic Mach numbers in the range 5 to 20 for various semi vertex angles of 20 degrees 25 degrees. It is seen that values have increase however, the trends are identical. The center of pressure remains in the range 50 to $55 \%$. The Mach number independent principle is clearly demonstrated here. As we know that when Mach number increases the shock wave angle decreases. But after a certain value of Mach number there is no decrease in the shock wave angle, which implies that initially with increase of Mach number the shock will on coming very close to the body but it will never collapse with the body.

\section{Conclusion}

Present theory demonstrates its application for a wide range of the Mach number, angle of attack and the semi vertex angle of the wedge. For semi vertex angle five to ten degrees the variation in the stiffness and damping derivatives is substantial, however; for large values of semi vertex angle the variation stiffness and damping derivative is only marginal. The variation in the center of pressure for stiffness and damping derivatives is from $20 \%$ to $55 \%$ for all the Mach numbers and semi vertex angle of the present study. The theory is valid only when the shock wave is attached with the nose of the wedge. In the present study the effect of Lee surface has been neglected as it is well known that the pressure on the lee surface will be negligible for hypersonic Mach numbers. The present theory could be handy at the initial design stage of the Aerospace Vehicles. Effects of viscosity \& wave reflection are also neglected in the present study. The present theory is simple and yet gives good results with remarkable computational ease with the error around ten percent.

\section{References}

[1] Sychev, V. V, Three Dimensional Hypersonic Gas Flow Past Slender Bodies at High Angles of Attack, Journal of Applied Mathematics and Mechanics, Vol. 24, August 1960, pp. 296- 306.

[2] Light Hill, M. J., Oscillating Aerofoil at High Mach Numbers, Journal of Aeronautical Sciences, Vol. 20, June 1953, pp. $402-406$.

[3] Appleton, J. P. , Aerodynamic Pitching Derivatives of a wedge in Hypersonic Flow, AIAA Journal, Vol. 2, November 1964, pp. 2034-2036.

[4] Mc Inthosh, S. C., Jr., Studies in Unsteady Hypersonic Flow Theory, Ph.D. Dissertation Stanford Univ., California, Aug. 1965.

[5] Hui, W.H., Stability of Oscillating Wedges and Caret Wings in Hypersonic and Supersonic Flows, AIAA Journal, Vol. 7, Aug. 1969, pp. 1524-1530.

[6] Ericsson, L. E., Viscous and Elastic Perturbation Effects on Hypersonic Unsteady Airfoil Aerodynamics, AIAA Journal , Vol. 15 Oct. 1977, pp. 1481-1490. 
[7] Orlik-Ruckemann, K. J., Stability Derivatives of Sharp Wedges in Viscous Hypersonic Flow, AIAA Journal, Vol. 4June 1966, pp. 1001-1007.

[8] Mandl, P., Effect of Small Surface Curvature on Unsteady Hypersonic Flow over an Oscillating Thin Wedge, C. A. S. I. Transactions, Vol. 4, No. 1, March 1971, pp. 47-57.

[9] Ghosh, Kunal, Hypersonic large deflection similitude for oscillating delta wings, Aeronautical Journal, Oct. 1984, pp. 357-361.

[10] Lui, D. D. and Hui W. H., Oscillating delta wings with attached shock waves, AIAA Journal, June 1977, 15, 6, pp. 804-812.

[11] Asha Crasta and Khan S. A., Oscillating Supersonic delta wing with Straight Leading Edges, International Journal of Computational Engineering Research, Vol. 2, Issue 5, September 2012, pp. 1226-1233, ISSN:2250-3005.

[12] Asha Crasta and Khan S. A., High Incidence Supersonic similitude for Planar wedge, International Journal of Engineering research and Applications, Vol. 2, Issue 5, September-October 2012, pp. 468-471, ISSN: 2248- 9622.

[13] Asha Crasta, M. Baig, S. A. Khan, Estimation of Stability derivatives of a Delta wing in Hypersonic flow, International Journal of Emerging trends in Engineering and Developments, Vol.6, Issue 2, September 2012, pp. 505-516, ISSN:2249-6149.

[14] Asha Crasta, S. A. Khan, Estimation of stability derivatives of Oscillating Hypersonic delta wings with curved leading edges, International Journal of Mechanical Engineering \& Technology, Volume 3, Issue 3, Dec 2012, pp. 483-492, ISSN 0976 - 6340 (Print), ISSN 0976 - 6359 (Online).

[15] Asha Crasta and S. A. Khan, Stability Derivatives in the Newtonian Limit, The International Journal of Advanced Research in Engineering and Technology, Volume 4, Issue 7, Nov-Dec 2013, pp. 276-289, ISSN - 6480 (Print), ISSN 0976 - 6499 (Online)

[16] S. A. Khan, Asha crasta, Oscillating supersonic delta wings with curved leading edges, International journal of Advanced studies in Contemporary Mathematics. Volume 20, No. 3, July 2010, pp. 359-372.

[17] Asha Crasta and S. A. Khan, Hypersonic Similitude for Planar Wedges, The International Journal of Advanced Research in Engineering and Technology, Volume 5, Issue 2, February 2014, pp. 16-31. ISSN: 0976 - 6480 (Print), ISSN: 0976 - 6499 (Online).

[18] Asha Crasta and Khan S. A., Stability derivatives of a delta wing with Straight leading edge in the Newtonian limit, International Journal of Engineering research and Applications, Volume 4, Issue 3, March 2014, pp. 32-38, ISSN: 2248-9622.

[19] Asha crasta and Khan S. A., Estimation of Stability Derivatives for a Planar Wedge in the Newtonian Limit, IOSR Journal of Mathematics (IOSR-JM), Volume 10, Issue 2, (March-April 2014), pp. 01- 06. 